ARTICLE

\title{
Activation mechanism of human soluble guanylate cyclase by stimulators and activators
}

Rui Liu (D) ${ }^{1,4}$, Yunlu Kang (10 ${ }^{1,4}$ \& Lei Chen (1) 1,2,3凶

Soluble guanylate cyclase ( $\mathrm{SGC}$ ) is the receptor for nitric oxide (NO) in human. It is an important validated drug target for cardiovascular diseases. SGC can be pharmacologically activated by stimulators and activators. However, the detailed structural mechanisms, through which $\mathrm{SGC}$ is recognized and positively modulated by these drugs at high spacial resolution, are poorly understood. Here, we present cryo-electron microscopy structures of human sGC in complex with NO and sGC stimulators, YC-1 and riociguat, and also in complex with the activator cinaciguat. These structures uncover the molecular details of how stimulators interact with residues from both $\beta \mathrm{H}-\mathrm{NOX}$ and $\mathrm{CC}$ domains, to stabilize sGC in the extended active conformation. In contrast, cinaciguat occupies the haem pocket in the $\beta$ $\mathrm{H}-\mathrm{NOX}$ domain and SGC shows both inactive and active conformations. These structures suggest a converged mechanism of sGC activation by pharmacological compounds.

\footnotetext{
${ }^{1}$ State Key Laboratory of Membrane Biology, College of Future Technology, Institute of Molecular Medicine, Beijing Key Laboratory of Cardiometabolic Molecular Medicine, Peking University, 100871 Beijing, China. ${ }^{2}$ Peking-Tsinghua Center for Life Sciences, Peking University, 100871 Beijing, China.

${ }^{3}$ Academy for Advanced Interdisciplinary Studies, Peking University, 100871 Beijing, China. ${ }^{4}$ These authors contributed equally: Rui Liu, Yunlu Kang.

凶email: chenlei2016@pku.edu.cn
} 
$\mathrm{N}$ itric oxide (NO) signaling plays an essential role in many physiological processes, and deregulation of $\mathrm{NO}$ signaling can lead to a spectrum of diseases ${ }^{1-4}$. NO signaling is initiated by the activation of $\mathrm{NO}$ synthase in the donor cell to produce NO molecules. NO then readily crosses target cell membranes and binds to its primary receptor soluble guanylate cyclase (sGC), which in turn boosts the activity of sGC several hundred-fold to produce intracellular cGMP2,5.

sGC is a heterodimer composed of one $\alpha$ and one $\beta$ subunit. The $\alpha$ and $\beta$ subunits share sequence homology and a common domain arrangement: the N-terminal H-NOX and PAS domains, the middle CC domain, and the C-terminal catalytic domain ${ }^{6}$. Recent high-resolution structures of human sGC in different functional states have revealed that $\mathrm{sGC}$ is an allosteric enzyme that is composed of three structural modules: the sensor module, the transducer module, and the catalytic module $^{7}$. Saturated NO binding to sGC drives the conformational change of the sensor module. This signal is conveyed to the adjacent transducer module and is further allosterically transmitted to the catalytic module to open the GTP binding cleft and enhance its activity ${ }^{7}$. The conformational changes within each module during sGC activation are associated with the large overall structural reconfiguration of the whole enzyme: inactive sGC has a bent shape, while active sGC is extended ${ }^{7}$. Similar structural changes were also observed in the medium-resolution structures of sGC from insect Manduca sexta ${ }^{8}$, further emphasizing the evolutionarily conserved structural mechanism of sGC activation by NO. The H-NOX domain of the $\beta$ subunit in the sensor module harbors a prosthetic ferrous haem $\left(\mathrm{Fe}^{2+}\right)$ moiety, which is essential for NO sensing 9 . In vitro structural and biochemical studies showed the oxidization of ferrous haem into ferric haem $\left(\mathrm{Fe}^{3+}\right)$ can trap sGC in the inactive state ${ }^{10-12}$. Moreover, the ferric haem can easily dissociate from sGC; this results in haem-free sGC, which has low activity and responds poorly to NO stimulation ${ }^{13}$. The haem oxidization and dissociation processes have been observed in vivo as well and can lead to the attenuation of NO signaling in several pathological conditions, such as in inflammation or under oxidative stress ${ }^{14,15}$.

To enhance the downstream of $\mathrm{NO}$ signaling by increasing the sGC activity in related diseases, two types of small pharmaceutical molecules have been developed, namely, sGC stimulators and activators. sGC stimulators can activate sGC with ferrous haem without NO, and also have strong synergistic activation effect with NO. Therefore, sGC stimulators can enhance downstream cGMP signaling both in the absence and in the presence of $\mathrm{NO}^{4,16,17}$. The sGC stimulator riociguat is clinically used for the treatment of pulmonary arterial hypertension and chronic thromboembolic pulmonary hypertension ${ }^{18}$, and another stimulator, vericiguat, was developed for the treatment of symptomatic chronic heart failure with reduced ejection fraction ${ }^{19}$. On the other hand, sGC activators can bind to and activate the haemoxidized and haem-free sGC irrespective of the upstream NO signaling. They are used to activate sGC where impaired NO signaling is caused by haem oxidization and the subsequent loss of haem in $\mathrm{sGC}^{16,17}$. Despite their functional importance, the structural mechanisms through which sGC stimulators and activators bind and activate sGC at high resolution have been unknown for decades, especially in the context of full-length human sGC. Here we investigated the structures of human $\alpha 1 \beta 1$ sGC in complex with the stimulator YC- 1 and riociguat and the activator cinaciguat by cryo-electron microscopy (cryo-EM). These structures not only reveal the detailed interactions involved in sGC-ligand recognition but also uncover the structural changes associated with drug binding and enzyme activation.

\section{Results}

Structure of sGC in complex with YC-1 or riociguat in the presence of NO. YC-1 is a prototype sGC stimulator ${ }^{20}$, and based on its chemical structure, a series of stimulators, such as BAY412272, BAY41-8543, riociguat (BAY 63-2521), and vericiguat (BAY 1021189), have been developed (Supplementary Fig. 1a). Among them, riociguat exhibits high potency, and good drug metabolism and pharmacokinetic properties and has been approved for clinical use $\mathrm{e}^{21}$. Previous studies suggested YC-1 might bind to the al H-NOX domain 22,23 , the catalytic module ${ }^{24-27}$, or the $\beta 1 \mathrm{H}-\mathrm{NOX}$ domain ${ }^{28-30}$. A recent cryo-EM map of M.sexta sGC at $5.8 \AA$ resolution in the presence of YC-1 and NO revealed an extra density located between the $\beta \mathrm{H}-\mathrm{NOX}$ and CC domains ${ }^{8}$. This extra density was suggested to be the putative $\mathrm{YC}-1$ molecule. However, the exact binding pose of $\mathrm{YC}-1$ and residues on sGC that interact with YC-1 could not be determined due to the limited resolution. Moreover, it is still questionable whether this site is the functional stimulatory site for human sGC, since no associated mutagenesis data were available. To directly identify the stimulator-binding site at high resolution, we embarked structural studies using cryo-EM. As the first step, we confirmed that purified human $\alpha 1 \beta 1$ sGC can be activated by the stimulator YC-1 and riociguat (Fig. 1 and Supplementary Fig. 1). The activation of sGC by stimulators and NO shows strong synergistic effects (Fig. 1b) ${ }^{31,32}$, suggesting that both the stimulators and NO promote sGC to adopt the same biochemically active state. Therefore, we prepared the cryo-EM sample of full-length human $\alpha 1 \beta 1$ sGC in complex with YC- 1 or riociguat in the presence of saturated $\mathrm{NO}$ donor. The final reconstruction reached a resolution of $3.9 \AA$ and $3.7 \AA$ in the presence of YC- 1 and riociguat, respectively (Fig. 1c-f, Supplementary Figs. 2 and 3 , and Supplementary Table 1).

The structures of sGC in complex with $\mathrm{NO}$ and stimulators show an extended conformation (Fig. 1c and d), similar to the NO-activated state, with a root-mean-square deviation (RMSD) of $0.8 \AA$ between the $\mathrm{NO}+\mathrm{YC}-1$ structure and the NO-activated state structure (PDB ID: 6JT2) 7 . We observed the haem-H105 bonds were broken in these structures (Supplementary Fig. $2 \mathrm{~m}$ ). The cryo-EM density maps revealed that YC-1 and riociguat bind at the same site on sGC, similar to the site observed in M.sexta $\mathrm{sGC}^{8}$. The stimulator-binding site is located between the $\beta$ $\mathrm{H}-\mathrm{NOX}$ and CC domains (Fig. 1e and $\mathrm{f}$ ), in agreement with their similar chemical structures. The local map qualities are sufficient for us to model YC-1 and riociguat molecules (Fig. 1e and f).

The stimulator-binding site. $\mathrm{YC}-1$ is clamped in the cleft between the $\mathrm{N}$ - and C-terminal subdomains of the $\beta 1 \mathrm{H}$-NOX and CC domains (Fig. 2). The side chain of $\beta 1$ Y112 stacks with the terminal phenyl ring of $\mathrm{YC}-1$, which also makes hydrophobic interactions with the side chain of $\beta 1$ Y2, F4, and haem (Fig. $2 \mathrm{a}$ and $\mathrm{d}$ ). The central indazole moiety of YC-1 interacts with Y83, F77, and F4 of the $\beta 1 \mathrm{H}-\mathrm{NOX}$ domain (Fig. $2 \mathrm{a}, \mathrm{b}$, and d). The furan group of YC- 1 interacts with $\beta 1 \mathrm{~V} 39, \beta 1 \mathrm{R} 40$, and $\alpha 1 \mathrm{~L} 425$ (Fig. $2 \mathrm{~b}$ and $\mathrm{d}$ ). The terminal hydroxyl group of $\mathrm{YC}-1$ makes hydrogen bonds with $\beta 1 \mathrm{E} 370$ on the CC domain (Fig. $2 \mathrm{~b}$ and d). Compared with $\mathrm{YC}-1$, the core of riociguat binds to sGC in a similar way (Fig. 2c and e), but the newly introduced diaminopyrimidine group makes additional polar interactions with $\beta 1$ S81 (Fig. $2 c$ and e) and the terminal methylcarbamate moiety is close to a1 R428 (Fig. 2c and e). We next examined the effects of riociguat on various stimulator-binding site mutants in the presence of saturated butyl isocyanide (BIC), which is a distal haem ligand and partial agonist for $\mathrm{sGC}^{33}$ (Fig. 2f and Supplementary Fig. 6i). We chose BIC instead of NO donors, because sGC can be 

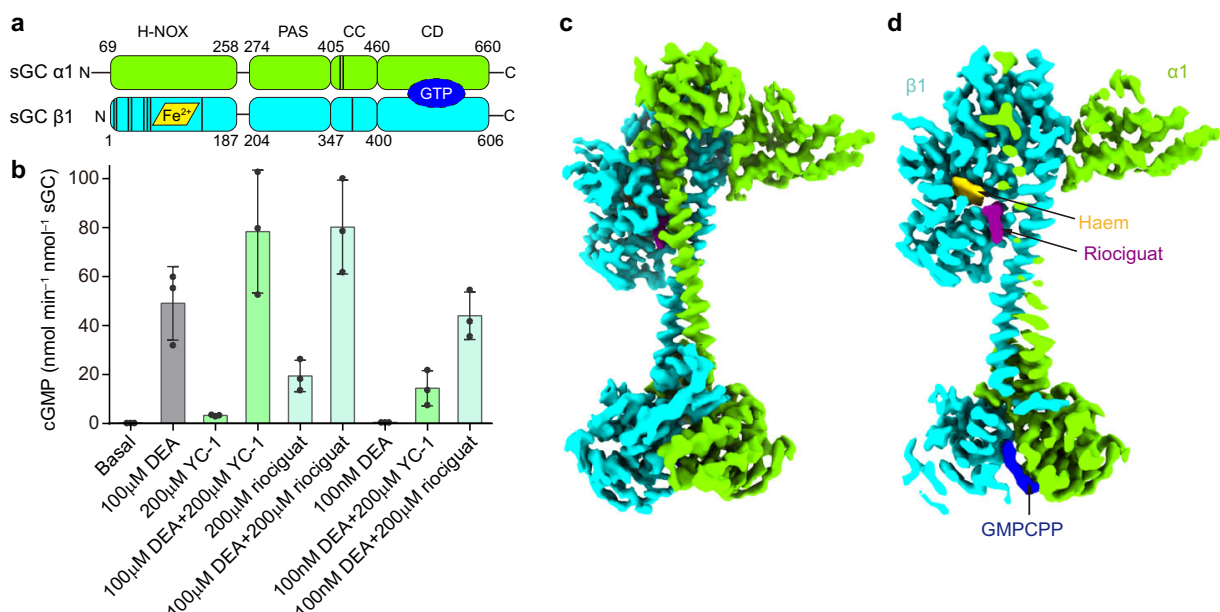

e
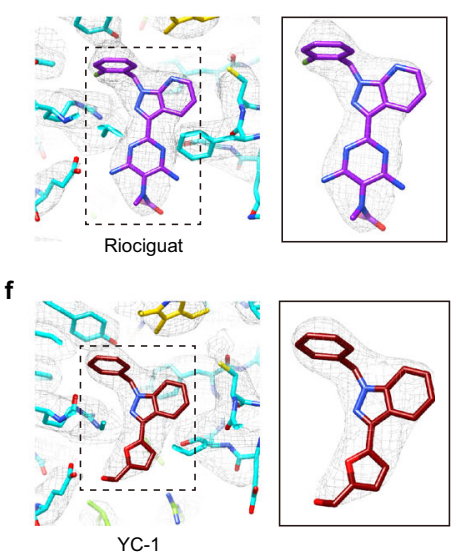

Fig. 1 Cryo-EM structure of sGC in the presence of NO and stimulators. a Domain organization of the human $\alpha 1 \beta 1$ sGC heterodimer. The haem cofactor and the GTP substrate-binding site are shown in yellow and blue, respectively. The positions of residues interacting with riociguat are indicated with vertical dark lines. $\mathbf{b}$ End-point activity assay of wild-type sGC in the presence or absence of DEA, YC-1, or riociguat as indicated. Mean \pm s.d., $n=3$ biologically independent reactions. Source data are provided as a Source Data file. c Cryo-EM map of sGC in complex with NO and riociguat. The densities of haem, riociguat, and GMPCPP are shown in yellow, purple, and blue, respectively. $\mathbf{d}$ The cut-open view of (c). e Density at the riociguat-binding site. The map is shown as a gray mesh and atomic model is shown as sticks. $\mathbf{f}$ Density at the YC-1-binding site. The map is shown as a gray mesh and atomic model is shown as sticks.

dose-dependently activated by riociguat in the presence of saturated $\mathrm{BIC}(1 \mathrm{mM})$ with an $\mathrm{EC}_{50}$ value of $20.08 \mu \mathrm{M}$ (Fig. $2 \mathrm{f}$ and Supplementary Fig. 6i) and BIC concentration in the reaction system is easy to control, while NO release from NO donors, such as DEA NONOate, heavily depends on $\mathrm{pH}$, temperature and incubation time and therefore NO concentration is difficult to control accurately, especially in an aerobic environment. We found mutations of F77, E370 and V39 on $\beta 1$ subunit, all of which interact with the common structural features of YC-1-type stimulators, into alanine residues reduced the potency of riociguat to different extent (Fig. $2 \mathrm{f}$ and Supplementary Table 2). Mutation of $S 81$ on the $\beta 1$ subunit that interacts with the tail of riociguat moderately reduced the potency of riociguat. Because S81 only interact with riociguat but not YC-1 (Fig. 2 d, e), we speculate the interaction between $\mathrm{S} 81$ and the tail of riociguat might play a role in the enhanced potency of riociguat compared with YC-1. BAY41-2272 and BAY41-8543 are additional YC-1-type sGC stimulators that can activate sGC with $\mathrm{EC}_{50}$ values at the micromolar range 23,34 (Supplementary Fig. 1a). Our structures suggest these stimulators bind sGC in a similar pose compared to riociguat and their distinct tails might dictate their different potency. In addition, we found mutations of L425A and R428A on a 1 subunit slightly increased the potency of riociguat. Because L425 and R428 are on the same side of the diaminopyrimidine group and close to the bulky diaminopyrimidinemethylcarbamate tail of riociguat (Fig. 2e), we speculate truncation of L425 and R428 to alanines would leave more space at this region so that riociguat might adopt a more favorable conformation to engage with other interacting residues on sGC. These data also suggest further structure-based modification of chemical groups on the tail of riociguat might result in improved molecules that bind sGC with higher potency.

By superimposing the sGC structure in complex with stimulators and $\mathrm{NO}$ onto the structure of the NO-activated state (PDB ID: 6JT2) (Fig. 2g) ${ }^{7}$, we found that there are several sterical clashes between the stimulator molecules and the $\beta 1 \mathrm{H}-\mathrm{NOX}$ domain of the NO-activated state (Fig. 2g). The binding of riociguat pushes $\alpha \mathrm{E}$ away from the stimulator-binding site. The Ca atom of Y83 shows a $1.0 \AA$ shift (Fig. 2g). Moreover, the rotamer conformations of several residues are altered as well. The phenyl ring of F4 swings away from the stimulator-binding site and the adjacent F74 shows a concomitant movement (Fig. 2g-i). These conformational changes collectively reshape the stimulator-binding pocket, which does not exist in the NOactivated structure, suggesting the stimulators bind sGC through an induced-fit mechanism. Furthermore, the stimulator binds beside the haem cofactor and might perturb the haem environment (Fig. 2a-c), which explains why YC-1 shifts the Soret peak positions of the sGC-CO complex ${ }^{35}$, changes the Fe-CO stretching frequency in the Raman spectrum ${ }^{28}$, and increases the affinity of CO for the M.sexta NT construct ${ }^{36}$. Because the stimulators bind to a site co-formed by the $\beta 1 \mathrm{H}-\mathrm{NOX}$ and CC domains (Figs. 1 and 2) which are spatially separated in the bent inactive conformation ${ }^{7}$, we propose the binding of stimulators facilitates the activating interactions between $\beta 1 \mathrm{H}-\mathrm{NOX}$ and CC domains and thus stabilizes sGC in the extended conformation for activation. This agrees with the positive cooperativity between the stimulators and NO. To further support this, we exploited the previously identified proline mutations in the hinge region of $\mathrm{CC}$ domains ( $\alpha 1$ D423P or $\beta 1$ G356P) that destabilize the long helices of the transducer module in the extended active conformation due to reduced main chain hydrogen bonding ${ }^{7}$. We reasoned that these mutations might inhibit the transmission of the NOactivation signal from sensor module to transducer module and therefore might elevate the free energy threshold for sGC activation and shift the inactive-active state equilibrium toward the inactive state. Indeed, although $\mathrm{NO}$ can bind onto these mutants $^{7}$, the activation effect of $\mathrm{NO}$ was impaired by these mutations (Fig. $2 \mathrm{k}$ and Supplementary Fig. 6j). Because stimulators bind sGC in the extended conformation, these mutants might allosterically reduce the binding of stimulators. Conversely, once bound, stimulators might stabilize the CC domain of these mutants in the extended conformation to some extent, since the positions of these proline mutations are either above or at the same level of the stimulator-binding site on sGC (Fig. 2j). We found riociguat alone failed to activate either $\alpha 1$ D423P or $\beta 1$ G356P mutant (Fig. 2k and Supplementary Fig. 6j), likely due to reduced binding of riociguat to these mutants. However, the simultaneous application of $\mathrm{NO}$ and riociguat markedly boosted their activity (Fig. 2k and Supplementary 
a

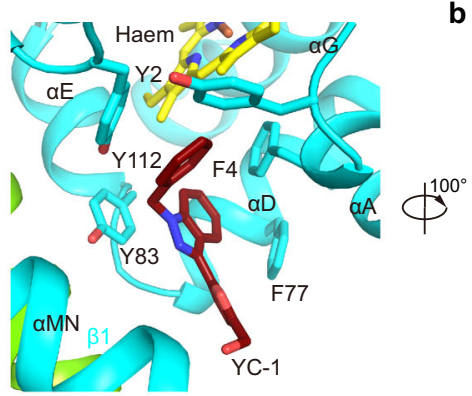

b

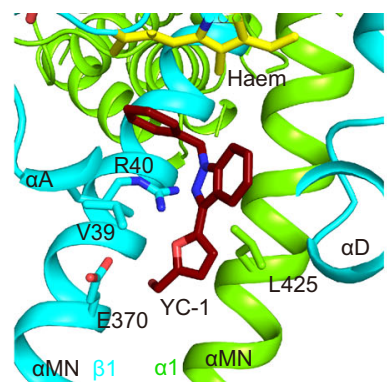

c

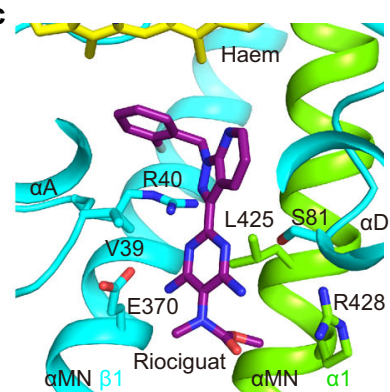

d

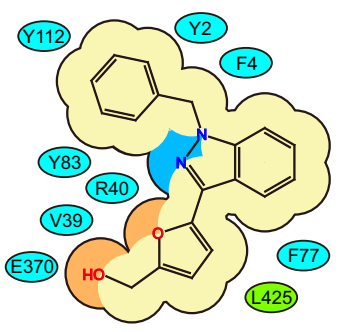

YC-1 e

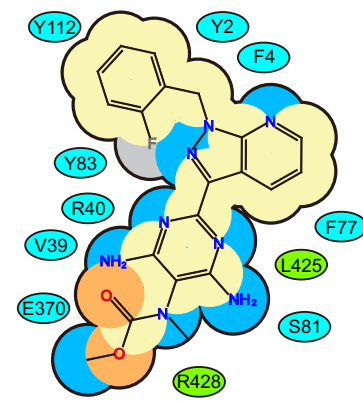

Riociguat $\mathbf{f}$

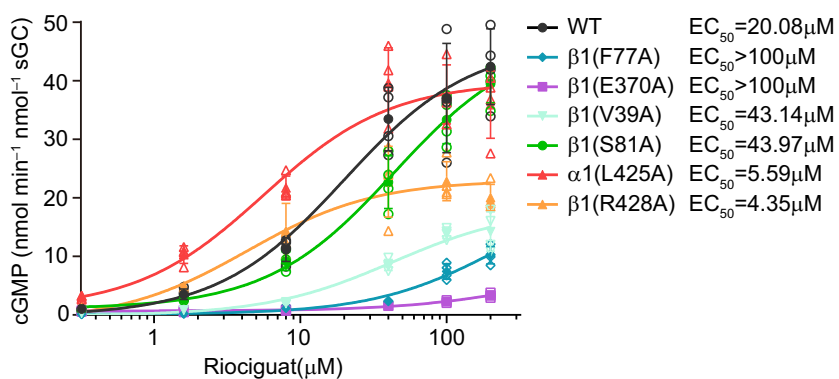

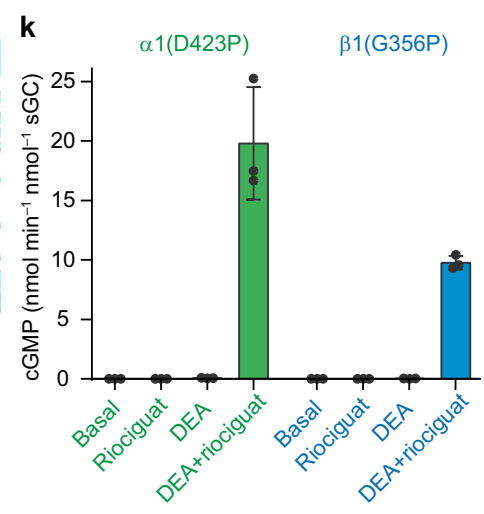

g

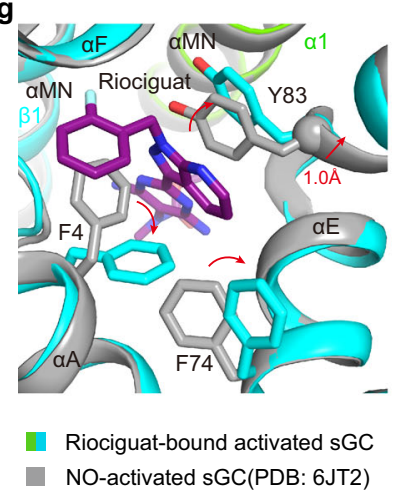

h

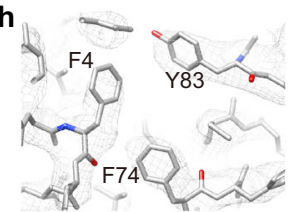

NO-activated SGC(PDB: 6JT2)

i

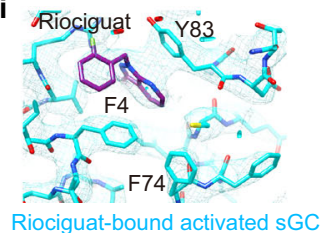

j

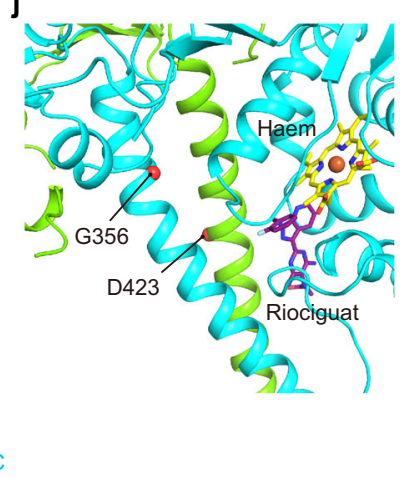

Fig. 2 Binding site of sGC stimulators. a The interactions between $\mathrm{YC}-1$ and sGC. Protein is colored the same as in Fig. 1. b A 100 rotated view compared to (a). c The interactions between riociguat and sGC in the same view as in (b). $\mathbf{d}$ Cartoon representation of interactions between YC-1 and sGC. Residues from the $\alpha 1$ and $\beta 1$ subunits are shown as green and cyan, respectively. $\mathbf{e}$ Cartoon representation of interactions between riociguat and sGC. Residues are colored the same as in (d). $\mathbf{f}$ Dose-dependent activation curve of wild type and alanine mutants of sGC in the presence of $1 \mathrm{mM}$ BIC and riociguat at different concentrations ranged from $0.32 \mathrm{mM}$ to $200 \mathrm{mM}$. Mean \pm s.d., $n=3$ or 4 independent reactions. Source data are provided as a Source Data file. g Structure comparison between NO- and riociguat- bound states (colored) and the NO-activated state (gray) at the stimulator-binding site. The conformational changes induced by riociguat binding are indicated by red arrows. $\mathbf{h}-\mathbf{i}$ Cryo-EM density maps around the sGC stimulators binding site in the NO-activated state (h) and NO- and riociguat- bound state (i). The residues undergo conformational changes in the two states shown in ( $\mathbf{g}$ ) are labeled. j Locations of the $\alpha 1 \mathrm{D} 423$ and $\beta 1 \mathrm{G} 356$ residues in the NO- and riociguat- bound state. The aC atoms of $\alpha 1 \mathrm{D} 423$ and $\beta 1 \mathrm{G} 356$ are shown as red spheres. k End-point activity assay of sGC proline mutations in the absence or presence of $100 \mathrm{mM}$ DEA or $200 \mathrm{mM}$ riociguat. Mean $\pm \mathrm{s} . \mathrm{d}$., $n=3$ independent reactions. Source data are provided as a Source Data file.

Fig. 6j), likely because the free energy contributed by simultaneous binding of $\mathrm{NO}$ and riociguat onto sGC overcomes the elevated free energy threshold for enzyme activation of these two mutants, further highlighting the strong synergistic effect of $\mathrm{NO}$ and riociguat.

Structure of sGC in complex with the activator cinaciguat. sGC activators, represented by cinaciguat (BAY58-2667) (Supplementary Fig. 6a), bind and activate haem-oxidized or haem-free $\mathrm{sGC}^{37}$. Although previous studies on the structure of cinaciguat in complex with H-NOX domain of symbiotic cyanobacteria Nostoc $s p$ provided valuable insights into the mechanism of cinaciguat binding ${ }^{38}$, the Nostoc $\mathrm{H}-\mathrm{NOX}$ has only $\sim 35 \%$ sequence identity to human sGC and its NO-binding properties are largely different from sGC either ${ }^{39}$. To elucidate the binding and activation mechanism of human sGC in the context of full-length enzyme, we prepared sGC protein in complex with cinaciguat through two approaches for cryo-EM studies: in the first approach, we oxidized ferrous sGC protein using NS2028 (8Bromo-1H,4H-[1,2,4] oxadiazolo[3,4-c][1,4]benzoxazin-1-one), a reagent that can oxidize ferrous haem to ferric haem ${ }^{11}$, and used cinaciguat to replace the oxidized haem (Fig. $3 \mathrm{a})^{40}$; in the second approach, we added cinaciguat to the purified haem-free sGC protein obtained by detergent treatment (Fig. 3b and Supplementary Fig. 4$)^{41}$. Unexpectedly, we found that not only the bent 

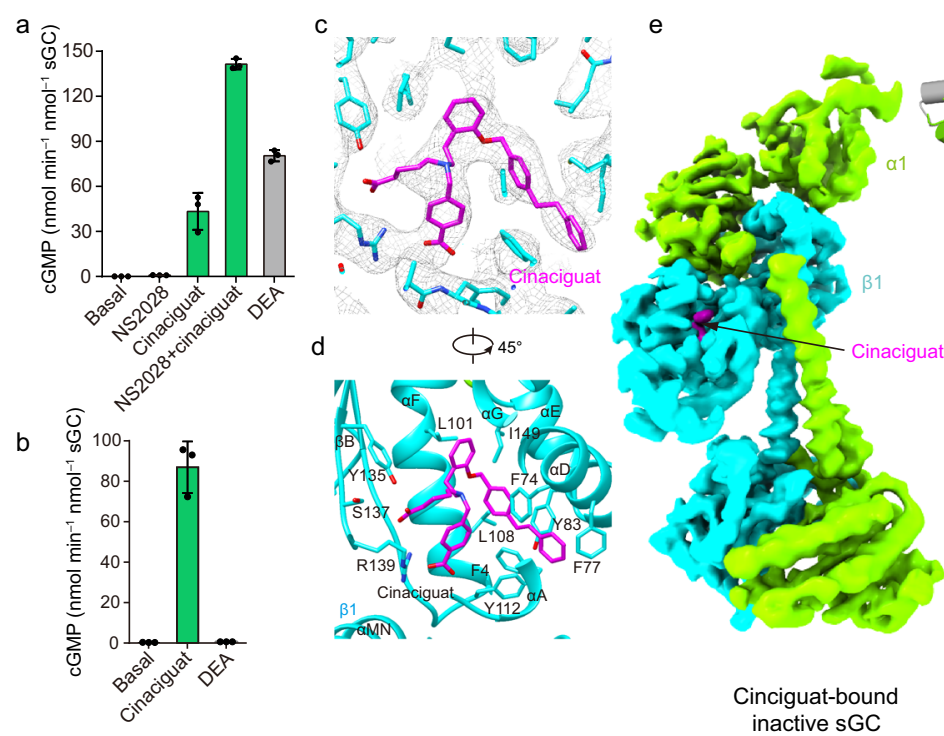

f

g

Fig. 3 Structure of $\mathbf{s G C}$ in the cinaciguat-bound inactive state. a End-point activity assay of the wild-type sGC samples in the presence or absence of $20 \mathrm{mM}$ NS2028, $20 \mathrm{mM}$ cinaciguat or $100 \mathrm{mM}$ DEA. NS2028 oxidizes the ferrous haem in sGC to ferric haem. Mean \pm s.d., $n=3$ biologically independent reactions. Source data are provided as a Source Data file. $\mathbf{b}$ End-point activity assay of the haem-free sGC samples with or without $20 \mathrm{mM}$ cinaciguat or $100 \mathrm{mM}$ DEA as indicated. Mean \pm s.d., $n=3$ biologically independent reactions. Source data are provided as a Source Data file. c Density at the cinaciguat binding pocket in the $\mathrm{Cl}$ state. Cinaciguat is shown as magenta sticks. $\mathbf{d}$ The interactions between cinaciguat and sGC in the $\mathrm{Cl}$ state. $\mathrm{A} 45^{\circ}$ rotated view compared to (c). e Cryo-EM map of sGC in the cinaciguat bound inactive $(\mathrm{Cl})$ state. The density of cinaciguat is shown in magenta. $\mathbf{f}$ Structural comparison of SGC in the $\mathrm{Cl}$ state (colored) and haem-oxidized state (gray) by aligning the $\beta 1 \mathrm{H}$-NOX domain. $\mathbf{g ~ A ~} 90^{\circ}$ rotated zoom-in view of the $\beta 1 \mathrm{H}-\mathrm{NOX}$ domain boxed in $\mathrm{f}$ (black rectangle). The side chains of $\mathrm{H} 105, \mathrm{Y} 112$, and $\mathrm{F} 4$ are shown as sticks. $\mathbf{h} \mathrm{A} 90^{\circ}$ rotated view of catalytic module boxed in $\mathrm{f}$ (red rectangle). The angle between the $\alpha \mathrm{Q}$ helices is shown.

but also the extended conformations existed in both cryo-EM samples, and therefore we merged these data for image processing (Supplementary Fig. 5). The cryo-EM reconstruction of bent sGC and extended sGC reached resolutions of $4.1 \AA$ and $3.8 \AA$ after consensus refinement and $3.9 \AA$ and $3.6 \AA$ at the N-lobe after multibody refinement (Supplementary Fig. 5). The map qualities were sufficient to locate the cinaciguat molecules inside the haem pocket of the $\beta 1 \mathrm{H}-\mathrm{NOX}$ domain in both maps (Supplementary Fig. 5).

The cinaciguat-bound inactive structure. The overall shape of the cinaciguat-bound bent structure is similar to that of the inactive state (PDB ID: 6JT1) ${ }^{7}$ (Fig. 3). Cinaciguat binds inside the haem pocket of the $\beta 1 \mathrm{H}$-NOX domain (Fig. $3 \mathrm{c}$ and d). The benzoic acid group of cinaciguat interacts with positively charged R139 and the carboxybutyl group of cinaciguat makes hydrogen bonds with Y135 and S137 (Fig. 3d). The two middle phenyl rings of cinaciguat interact with hydrophobic residues in the haem pocket, including I149, L101, L108, and F74 (Fig. 3d). The terminal benzyl group of cinaciguat points to the outside of sGC and is surrounded by the aromatic F4 on $\alpha \mathrm{A}, \mathrm{F} 77$ on $\alpha \mathrm{D}, \mathrm{Y} 83$ on the $\alpha \mathrm{D}-\alpha \mathrm{E}$ loop, and $\mathrm{Y} 112$ on $\alpha \mathrm{F}$ (Fig. 3d). In comparison with the inactive sGC structure (PDB ID: 6JT1) ${ }^{7}$ (Fig. 3f), the replacement of haem by cinaciguat causes rotamer changes of F4 and Y112 which further leads to small but noticeable structural shifts of $\alpha \mathrm{F}$ and $\beta \mathrm{A}$ (residues 100-117) and a slightly altered conformation of the sensor module (Fig. 3g). These changes were amplified by the transducer module into a displacement of the catalytic module as a rotation of about $3.8^{\circ}$ (Fig. $3 \mathrm{~h}$ ). However, the GTP-binding site in the catalytic module is still incompatible with substrate binding and is devoid of substrate analog GMPCPP density (Supplementary Fig. 6b), confirming that this structure represents an inactive state. We name this structure as the cinaciguat-bound inactive (CI) state, in which the activator is bound but does not exert its activating function.

The cinaciguat-bound activated structure. In the cinaciguatbound extended conformation (Fig. 4a and b), cinaciguat interacts with a similar set of residues compared to the CI state (Fig. 4c and d). However, it induces a much larger conformational change of the $\beta 1 \mathrm{H}$-NOX domain and leads to the complete extension of sGC (Fig. 4). The RMSD between this structure and the NOactivated structure (PDB ID: 6JT2) is $0.39 \AA$. Moreover, we found the GMPCPP density inside the catalytic module (Fig. $4 \mathrm{~b}$ ), further confirming this is a catalytically active state. Therefore, we name this structure as the cinaciguat-bound activated (CA) state. To understand why cinaciguat-bound sGC can adopt both bent and extended structures, we compared the conformations of cinaciguat in the structures of the H-NOX domain in CI and CA states and the crystal structure of Nostoc H-NOX in complex with cinaciguat (PDB ID: 3L6J) (Fig. $4 \mathrm{f}-\mathrm{i})^{38}$. We found the interactions between the cinaciguat core and the $\beta 1 \mathrm{H}-\mathrm{NOX}$ domain are similar in these structures but the conformations of the terminal benzyl group of cinaciguat are dramatically distinct (Fig. 4f-i). This is consistent with the high flexibility of cinaciguat, which has 17 rotatable bonds, and multiple conformations of cinaciguat were previously observed in the NMR structures of the cinaciguat-bound isolated H-NOX domain (PDB ID: 5MNW) (Supplementary Fig. 6c). Although the conformation of $\beta 1$ H-NOX domain and cinaciguat molecule in both $\mathrm{CI}$ and CA states were different from Nostoc H-NOX structure (Supplementary Fig. 6e, f), the cinaciguat binds to conserved hydrophilic residues that are involved in haem binding, such as Y135, S137 and R139. However, there are several non-conserved residues between human sGC and Nostoc H-NOX domain which determine the conformational difference between them. In the Nostoc 
a

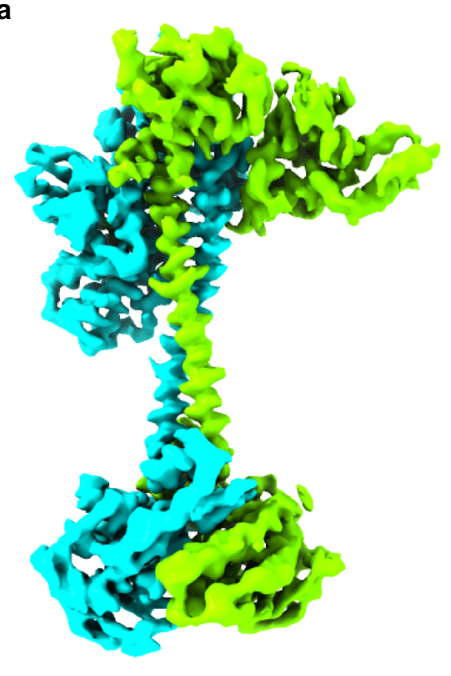

b

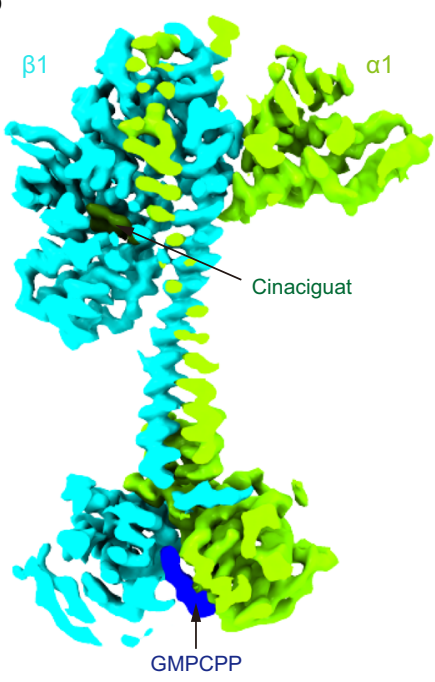

c
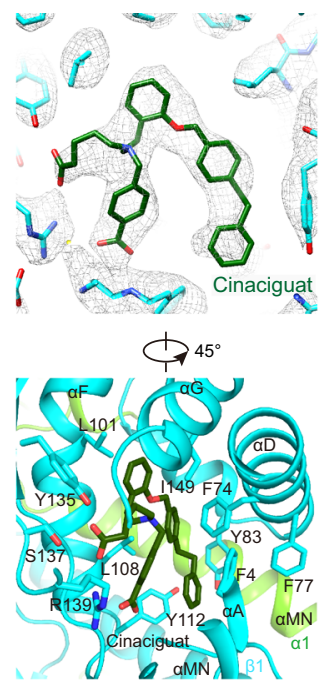

e

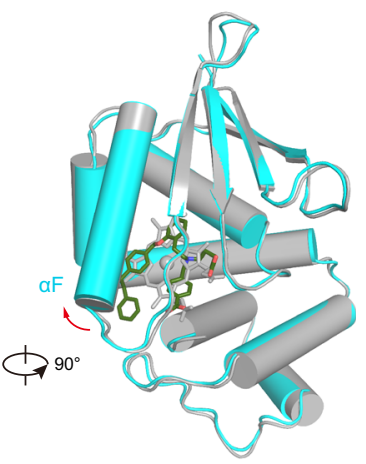

Cinciguat-bound activated sGC - NO-activated SGC(PDB: 6JT2)

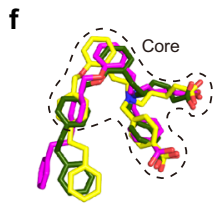

g

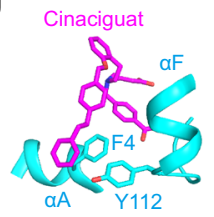

Inactive state

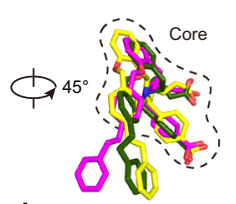

h

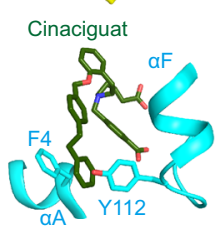

Active state
Nostoc(PDB ID: 3L6J)

Cinaciguat-bound inactive sGC Cinaciguat-bound activated sGC i

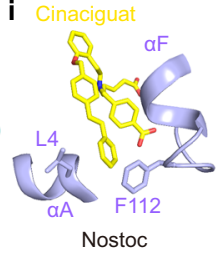

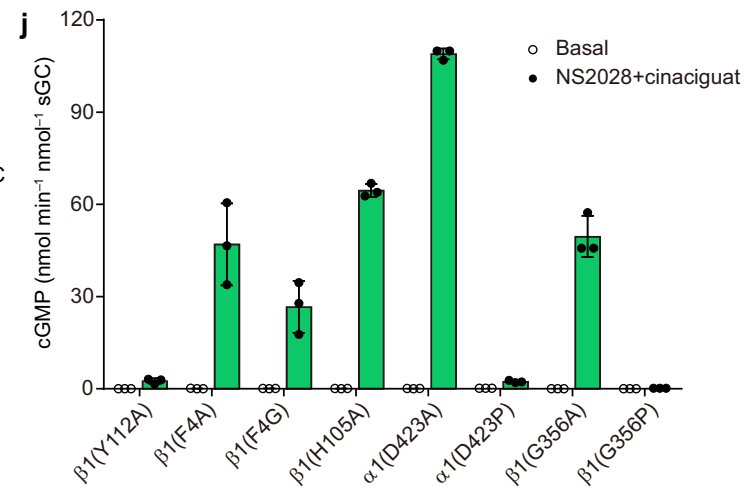

Fig. 4 Structure of sGC in the cinaciguat-bound activated state. a Cryo-EM map of sGC in the cinaciguat-bound activated state (CA) state. The density of cinaciguat is shown in green. $\mathbf{b}$ The cut-open view of (a). c Density at the cinaciguat-binding site in CA state. Cinaciguat is shown as dark green sticks. d The interactions between cinaciguat and sGC. A $45^{\circ}$ rotated view compared to (b). e Structure comparison of sGC $\beta 1 \mathrm{H}-\mathrm{NOX}$ in the CA state (colored) and the NO-activated state (gray). f Structural comparison of cinaciguat in the Nostoc H-NOX domain (PDB ID: 3L6J, yellow), Cl state (magenta) and CA state (green). The core of cinaciguat is indicated by dashes. $\mathbf{g}$ The interactions between the terminal benzyl group of cinaciguat and F4 and Y112 of sGC in the $\mathrm{Cl}$ state. $\mathbf{h}$ The interactions between the terminal benzyl group of cinaciguat and F4 and $\mathrm{Y} 112$ of sGC in the CA state. $\mathbf{i}$ The interaction between the terminal benzyl group of cinaciguat and L4 and F112 of the Nostoc H-NOX domain (PDB ID: 3L6J). $\mathbf{j}$ End-point activity assay of the various sGC mutants in the presence or absence of $20 \mathrm{mM}$ NS2028 and 20 mM cinaciguat as indicated. Mean \pm s.d., $n=3$ biologically independent reactions. Source data are provided as a Source Data file.

H-NOX structure, the hydrophobic benzyl group is bound by Y2, F112, and Y83 (Supplementary Fig. 6d), while in the CI and CA structures, the benzyl group makes additional interactions with F4 on $a \mathrm{~A}$ of human sGC (Fig. $4 \mathrm{~g}$ and $\mathrm{h}$ ), whereas a smaller leucine residue at this position in Nostoc $\mathrm{H}$-NOX replaces the F4 of the human counterpart (Fig. 4i). Between the CI and CA structures, F4 and Y112 have the largest conformational differences (Fig. 4g and h). To further explore the functions of F4 and Y112 on cinaciguat activation, we mutated these bulky residues into smaller residues. We found mutations of F4 into alanine or glycine moderately reduced the efficacy of cinaciguat-bound sGC, while mutation of Y112A completely abolished the activation by cinaciguat (Fig. 4j). Because Y112 is located on the C terminus of $\alpha \mathrm{F}$, which is important for sGC activation, we propose that cinaciguat in the CA state pushes the $\mathrm{C}$ terminus of $\alpha \mathrm{F}$ to induce a large conformational change of the sensor module and this signal is transmitted to catalytic module via the central transducer module, which finally leads to the full activation of the whole enzyme. In agreement with this model, cinaciguat failed to activate the $\alpha 1 \mathrm{D} 423 \mathrm{P}$ or $\beta 1 \mathrm{G} 356 \mathrm{P}$ mutants of the CC domain (Fig. $4 \mathrm{j}$ and Supplementary Fig. 6g), due to the destabilizing effect of proline mutation at the hinge region of the transducer module ${ }^{7}$. In contrast, cinaciguat in the CI state does not push Y112 far enough for a large conformational change of the sensor module (Fig. 3g), and sGC still adopts an inactive structure. We further showed that cinaciguat could robustly activate the H105A mutant (Fig. 4j), suggesting H105 is not essential for cinaciguat activation. This is different from the activation by NO, which absolutely requires $\mathrm{H} 105$ for activation ${ }^{42,43}$. It is reported that extension of the terminal group of cinaciguat resulted in compound 20 with higher efficacy ${ }^{44}$ (Supplementary Fig. 6a), and Y112A reduced the activation effect of compound $20^{44}$, further highlighting the importance of $\mathrm{Y} 112$ for sGC activation by cinaciguat-type activators.

\section{Discussion}

It is well established that $\mathrm{NO}$ concentrations are perceived by sGC and converted to cGMP levels for downstream signaling. How sGC senses NO is a fundamental question in the field. It is proposed that stoichiometric NO can partially activate the enzyme, while complete activation of $\mathrm{sGC}$ requires excess $\mathrm{NO}^{5}$. Recent small angle $\mathrm{X}$-ray scattering results provided low-resolution 
structural evidence that stoichiometric NO induces a partially active conformation which is in between the bent and the fullyextended conformation ${ }^{8}$, while excess NO renders sGC to predominately adopt the fully-extended conformation ${ }^{7}$. The stimulators, exemplified by the YC-1, can boost the activity of sGC in stoichiometric NO state to the level close to excess NO state ${ }^{8}$. In agreement with this, stimulators (YC-1 and riociguat) markedly enhanced the activity of sGC at low concentration of NO donor with positive co-cooperativity (Fig. 1b). But how this cooperativity is achieved at atomic level was enigmatic. Recent mediumresolution structure of M.sexta sGC provided the first clue of where $\mathrm{YC}-1$ binds $^{8}$. Our current studies provide near-atomic resolution depiction of how stimulators are recognized by sGC. The stimulators bind at a unique site that is formed by residues not only from the $\beta 1$ H-NOX domain of sensor module but also from the transducer module, and stabilize sGC in the extended conformation, intuitively explaining their positive cooperativity with NO. Notably, in order to obtain a homogenous sGC population for structural studies, we saturated sGC protein with excess NO in these cryo-EM samples. But we reasoned the stimulators also bind at the same site in the presence of stoichiometric NO, partial agonist CO or BIC, because on one hand, these ligands showed similar positive cooperativity with stimulators, and on the other hand, mutations in stimulator-binding pocket observed here altered the potency of stimulator in the presence of partial agonist, exemplified by BIC. Unfortunately, due to the small size of NO molecule (only two light atoms) and limited resolutions of the cryo-EM maps we obtained, we cannot directly visualize the densities of NO molecules. However, we observed the shift of Soret peak, markedly enhance sGC activity of our cryo-EM sample and the broken of H105-haem bonds in our cryo-EM maps, suggesting the haem was bound with $\mathrm{NO}$ and in 5 -coordination state in these structures. The exact binding sites of $\mathrm{NO}$ and the structure of sGC in stoichiometric NO state still await further investigation. The conformational changes in the sensor module and the transducer module induced by agonists, stimulators and activators are finally conveyed to the catalytic module via the inter-module connections to open the GTP substrate binding pocket. In addition to the YC-1 type stimulators, there are other sGC stimulators with distinct chemical structures $^{16}$, which might bind sGC at different sites. As some mutations in the catalytic module can enhance the sGC activity 24,45 , it is possible that certain hypothetic molecules can bind inside the catalytic module to open the substrate binding pocket and to activate sGC. Because the conformation of catalytic module is allosterically coupled to the structure of the sensor module ${ }^{46-48}$, these hypothetic molecules might likely induce similar extended conformation of sGC.

In contrast to stimulators, cinaciguat activator binds inside the haem pocket and replaces haem. It is striking that sGC molecules in both CA and CI conformation co-exist in the cinaciguat-bound sGC sample. But it is unknown if the sGC molecules in the CI state were trapped in the inactive conformation or they are in thermodynamic equilibrium with the CA state. Nevertheless, our structures suggest that modifications of the chemical groups of activators might yield next generation activators that can push Y112 or $\alpha \mathrm{F}$ more effectively, can increase the population of sGC in the CA state and thus can enhance the efficacy of activators.

In summary, the structures presented here reveal the molecular details of how human $\alpha 1 \beta 1$ sGC recognizes pharmaceutical YC1-type stimulators and cinaciguat-type activators, both of which bind outside of the catalytic module but can allosterically activate the enzyme (Fig. 5). These insights are likely instrumental to future design and optimization of therapeutic drugs that positively modulate the activity of sGC. The structures further strengthen the correlation between the extended conformation of
sGC and high enzymatic activity, and also suggest that the bentto-extended conformational change is a converged activation mechanism for sGC.

\section{Methods}

Cell culture. Sf9 or Sf 21 insect cells (Thermo Fisher Scientific) were cultured in SIM SF (Sino Biological) at $27^{\circ} \mathrm{C}$. The cell lines were routinely checked to be negative for mycoplasma contamination but have not been authenticated.

Protein expression and purification. To prepare protein sample for cryo-EM studies, supernatants of sf 9 or sf2 1 cells expressing wild-type sGC were loaded onto $2 \mathrm{ml}$ Streptactin Beads 4 FF (Smart-Lifesciences) and washed with W1 buffer (20 mM Tris, $\mathrm{pH} 8.0,500 \mathrm{mM} \mathrm{NaCl}, 2 \mathrm{mM}$ DTT) and W2 buffer(20 mM Tris, pH 8.0, $50 \mathrm{mM} \mathrm{NaCl}, 2 \mathrm{mM} \mathrm{DTT})$ at $4{ }^{\circ} \mathrm{C}$. Protein was eluted with elution buffer ( 50 mM Tris, $\mathrm{pH} 8.5,50 \mathrm{mM} \mathrm{NaCl}, 5 \mathrm{mM}$ desthiobiotin, 1 mM EDTA, 2 mM DTT) at $4{ }^{\circ} \mathrm{C}$. The eluate was digested with PreScission protease to remove GFP tag and further purified with a $1 \mathrm{ml}$ HiTrap Q HP column (GE Healthcare) and Superdex 200 Increase (GE Healthcare). For haem-free sGC, fractions eluted from the HiTrap Q HP column (GE Healthcare) were pooled, 10\% Tween 20 (final concentration) and PreScission protease were added to the protein, and samples were incubated at $4{ }^{\circ} \mathrm{C}$ for about $20 \mathrm{~h}$ to remove the haem and GFP tag from the protein. Then the mixture was diluted with buffer A (20 mM Tris, pH 8.0, 2 mM DTT) and then loaded onto a $1 \mathrm{ml}$ HiTrap Q HP column (GE Healthcare) again to separate the protein from impurities. The protein was eluted with buffer $\mathrm{B}(20 \mathrm{mM}$ Tris, $\mathrm{pH}$ $8.0,500 \mathrm{mM} \mathrm{NaCl}, 2 \mathrm{mM}$ DTT) at $4{ }^{\circ} \mathrm{C}$ in a linear gradient using the AKTA pure system (GE Healthcare). The peak fractions corresponding to the non-tag haemfree sGC were pooled and further purified by Superdex 200 increase (GE Healthcare). The peak fractions containing the sGC were analyzed by $12 \%$ SDSPAGE and were concentrated before flash frozen in liquid nitrogen. The UV-vis spectrums were measured by a spectrometer (Pultton).

To prepare protein sample for enzymatic activity assay, the coding sequence of the sGC $\alpha 1$ subunit tagged with C-terminal GFP-strep and $\beta 1$ subunit were cloned into the pFastBacl expression vectors, respectively. The constructs carrying point mutations were generated with Quick Change and corresponding baculoviruses were generated using the Bac-to-Bac system. Two baculoviruses for both $\alpha 1$ and $\beta 1$ subunits were co-infected into sf 9 cells for protein expression. Wild-type sGC and mutants were purified similarly as described above except ion-exchange chromatography was omitted. Protein concentrations were estimated using A280, with molar extinction coefficient of $83.2 \mathrm{mM}^{-1} \mathrm{~cm}^{-1}$. Haem concentrations were calculated using A431, with molar extinction coefficient of $111 \mathrm{mM}^{-1} \mathrm{~cm}^{-1,49}$. Haem content was the molar ratio between haem and protein.

Enzymatic activity assay. The purified haem-free sGC were diluted with $20 \mathrm{mM}$ triethanolamine (TEA pH7.6), $300 \mathrm{mM} \mathrm{NaCl}$ and subjected to the enzyme activity assay. The reaction system contained $14 \mathrm{nM}$ sGC, $60 \mathrm{mM}$ TEA (pH 7.6), $150 \mathrm{mM}$ $\mathrm{NaCl}, 0.5 \mathrm{mM}$ DTT, $5 \mathrm{mM} \mathrm{MgCl}{ }_{2}, 200 \mu \mathrm{M}$ GTP and various chemical drugs as indicated in a final volume of $20 \mu \mathrm{l}$. The final concentration of DMSO was $1 \%(\mathrm{v} / \mathrm{v})$. The assay mixture was incubated at $25^{\circ} \mathrm{C}$ for $10 \mathrm{~min}$, and then $80 \mu \mathrm{l} 125 \mathrm{mM}$ $\mathrm{Zn}(\mathrm{OAc})_{2}$ and $100 \mu \mathrm{l} 125 \mathrm{mM} \mathrm{Na}_{2} \mathrm{CO}_{3}$ were added to stop the reaction. The GTP$\mathrm{ZnCO}_{3}$ precipitation was removed by centrifuged at $17,000 \mathrm{~g}$ for $5 \mathrm{~min}$, and the supernatants were used for the quantification of cGMP with the cGMP ELISA Kit (Cayman Chemical) according to the instructions. For activity assay in Figs. 3a, b and $4 \mathrm{j}, 0.5 \mathrm{mM}$ DTT in the assay mixture was omitted and the reaction temperature was changed to $37^{\circ} \mathrm{C}$. Each assay was repeated for at least three independent reactions.

Cryo-EM sample preparation and data collection. The purified sGC was concentrated to $\mathrm{A}_{280}=4.5$ with an estimated concentration of $54 \mu \mathrm{M}$. For the sGC in complex with NO and YC-1, $200 \mu \mathrm{M}$ YC-1 (TargetMol), $1 \mathrm{mM}$ GMPCPP (Biorbyt), $1 \mathrm{mM}$ DEA NONOate (Cayman Chemical), $5 \mathrm{mM} \mathrm{MgCl}_{2}$ and $0.5 \mathrm{mM}$ fluorinated octyl-maltoside (FOM, Anatrace) were added to the wild type sGC. For the sGC in complex with $\mathrm{NO}$ and riociguat, $200 \mu \mathrm{M}$ riociguat (TargetMol), $1 \mathrm{mM}$ GMPCPP, $1 \mathrm{mM}$ DEA NONOate, $5 \mathrm{mM} \mathrm{MgCl}$, $0.5 \mathrm{mM}$ FOM were added to the wild type sGC. For the cinaciguat-bound sGC, two approaches were used for the sample preparation: in the first approach, the wild type sGC with ferrous haem were incubated with $200 \mu \mathrm{M}$ NS2028 and $200 \mu \mathrm{M}$ cinaciguat (TargetMol) at room temperature overnight, and then $1 \mathrm{mM}$ GMPCPP, $5 \mathrm{mM} \mathrm{MgCl}, 0.5 \mathrm{mM}$ FOM were added; in the second approach, the purified haem-free $\mathrm{sGC}$ was concentrated to $\mathrm{A}_{280}=4.5$, and then $200 \mu \mathrm{M}$ cinaciguat, $1 \mathrm{mM} \mathrm{GMPCPP}, 5 \mathrm{mM} \mathrm{MgCl}_{2}, 0.5 \mathrm{mM}$ FOM were added at $4{ }^{\circ} \mathrm{C}$. Protein samples were loaded onto glow-discharged Quantifoil 0.6/1 holey carbon gold grids and plunged into liquid ethane by Vitrobot Mark IV (Thermo Fisher Scientific). Cryo-grids were screened on a Talos Arctica electron microscope (Thermo Fisher Scientific) operating at $200 \mathrm{kV}$ using a Ceta $16 \mathrm{M}$ camera (Thermo Fisher Scientific). The screened grids were transferred to a Titan Krios electron microscope (Thermo Fisher Scientific) operating at $300 \mathrm{kV}$ with an energy filter set to a slit width of $20 \mathrm{eV}$. Images were recorded using a K2 Summit direct electron camera (Gatan Inc) in super-resolution mode at a nominal magnification of $\times 130,000$, corresponding to a calibrated super-resolution 


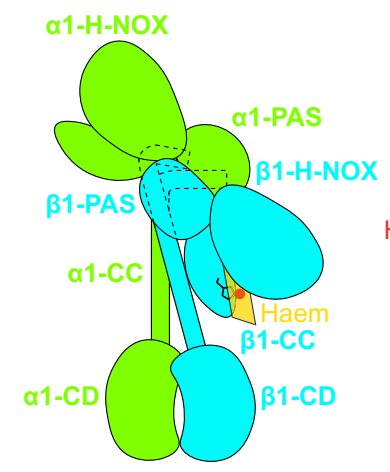

Haem-unliganded state

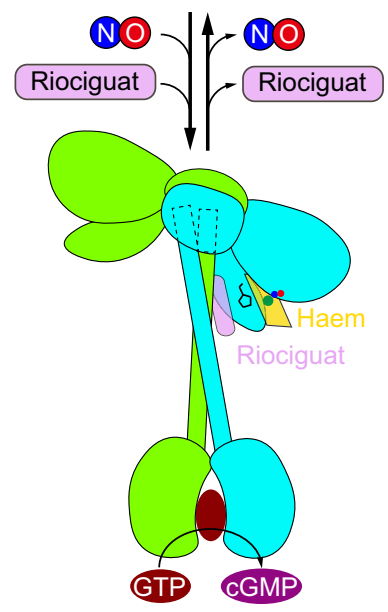

NO-stimulator-activated state

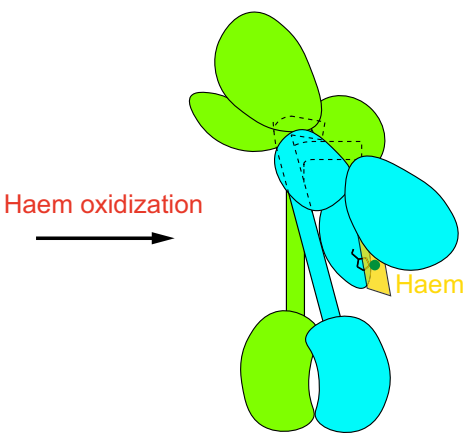

Haem-oxidized state

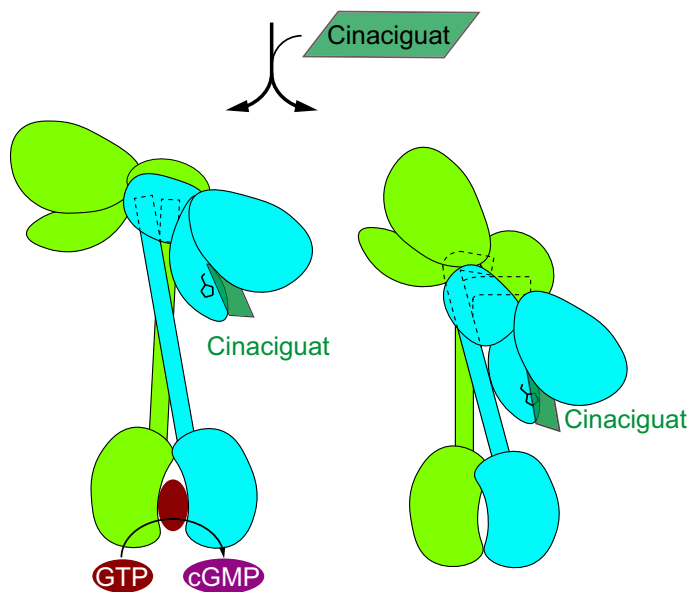

Cinaciguat-bound activated $(\mathrm{CA})$ state
Cinaciguat-bound inactive $(\mathrm{Cl})$ state

Fig. 5 Activation mechanism of sGC by stimulators and activators. Cartoon models of the SGC structures at different functional states. The colors of each individual subunit are the same as Fig. 1a. In the presence of NO, YC-1 type stimulators bind onto sGC with ferrous haem, between $\beta 1 \mathrm{H}-\mathrm{NOX}$ domain and CC domains, to stabilize SGC in the extended active conformation. Cinaciguat bind onto SGC at the haem-oxidized state or haem-free state, inside the $\beta 1$ $\mathrm{H}-\mathrm{NOX}$ domain, and sGC shows both extended active conformation and bent inactive conformation.

pixel size of $0.5225 \AA$. The defocus range was set from $-1.5 \mu \mathrm{m}$ to $-1.8 \mu \mathrm{m}$. Each image was acquired as a $7.68 \mathrm{~s}$ movie stack ( 32 frames) with a dose rate of $6.25 \mathrm{e}^{-} \AA^{-2} \mathrm{~s}^{-1}$, resulting in a total dose of about $48 \mathrm{e}^{-} \AA^{-2}$. All data acquisition was done using SerialEM.

Cryo-EM image analysis. The data processing workflows are shown in Supplementary Figs. 2, 3, 5 and Supplementary Table 1. Super-resolution movie stacks were gain-corrected, motion-corrected, mag-distortion corrected, dose-weighted, and binned to a pixel size of $1.045 \AA$ by MotionCor2 1.1 .0 using $5 \times 5$ patches $^{50}$ Contrast transfer function (CTF) parameters were estimated from non-doseweighted micrographs using Gctf v1.06 ${ }^{51}$. Micrographs with ice or ethane contamination, empty carbon, and poor CTF fit $(>5 \AA)$ were manually removed. All classification and reconstruction were performed with Relion $3.0^{52}$ unless otherwise stated. Particles were picked using Gautomatch (developed by Kai Zhang) and subjected to reference-free $2 \mathrm{D}$ classification to remove bad particles. For YC-1 dataset and riociguat dataset, previous human $\alpha 1 \beta 1 \mathrm{sGC}$ map in NO-activated state (EMD-9885) was low-passed to $30 \AA$ and used as initial model for 3D classification. For cinaciguat dataset, four initial models were generated using cryoSPARC ${ }^{53}$ and were used for multi-reference 3D classification in Relion 3.0. The particles selected from good 3D classes were re-centered and re-extracted, and their local CTF parameters were individually determined using Gctf $\mathrm{v} 1.06^{51}$. These particles were further refined using Relion 3.0 for consensus refinement. We further divided the whole molecule into two bodies-the larger "N lobe" and the smaller " $\mathrm{C}$ lobe" - for further multibody refinement in Relion 3.0. The FSC-based resolutions of each iteration during multibody refinement were monitored and we found the first iteration produced best resolution and map features (Supplementary Fig. 2d), probably due to the small size of each body and thus over-fitting during more iterations of multibody refinement. Therefore, we continued the first iteration with "-force_converge" option to generate the final maps of multibody refinement. The two half-maps of each lobe generated by $3 \mathrm{D}$ multibody refinement were subjected to post-processing in Relion 3.0. The masked and sharpened maps of each lobe were aligned to the consensus map using UCSF Chimera and summed together to generate the composite map for visualization and interpretation. All of the resolution estimations were based on the Fourier shell correlation (FSC) of 0.143 cutoff after correction of the masking effect ${ }^{54}$. B-factors used for map sharpening were automatically estimated by the post-processing procedure in Relion 3.0. Notably, for cinaciguat-bound dataset, images collected from samples produced by both approaches were found to be similar after initial processing, and therefore were combined for image processing workflow shown in supplementary Fig. 5.

Model building. The models of human $\alpha 1 \beta 1 \mathrm{sGC}^{7}$ (NO-activated state PDB ID: 6JT2 and heme-oxidized state PDB ID: 6JT1) were placed into the corresponding composite maps using UCSF chimera ${ }^{55}$ and manually rebuilt in $\operatorname{Coot}^{56}$. The composite maps were then converted into mtz files and the models were further refined by Phenix ${ }^{57}$ in reciprocal space, and phenix.real_space_refine ${ }^{57}$ and Coot in real space. The resolution of sGC cryo-EM map in riociguat-bound state was not sufficient to explicitly determine the location of the $\mathrm{F}$ atom on the phenyl ring, and the $\mathrm{F}$ atom was modeled according to the local chemical environment. Figures were made by Chimera $\mathrm{X}^{58}$, UCSF chimera and Pymol.

Quantification and statistical analysis. Global resolution estimations of cryo-EM density maps are based on the 0.143 FSC criterion ${ }^{54}$. The local resolution was estimated using Relion $3.0^{52}$. The number of independent reactions $(\mathrm{N})$ and the relevant statistical parameters for each experiment (such as mean or standard deviation) are described in the figure legends. No statistical methods were used to pre-determine sample sizes.

Reporting summary. Further information on research design is available in the Nature Research Reporting Summary linked to this article.

\section{Data availability}

The cryo-EM map of SGC in complex with YC1, riociguat and cinaciguat are accessible through the EMDB codes EMD-30619, EMD-30618, EMD-30620 and EMD-30621. The atomic coordinates have been deposited in the Protein Data Bank (PDB) with accession 
codes 7D9S, 7D9R, 7D9T and 7D9U. PDB entries used in this study are available in the PDB database under accession codes 6JT1, 6JT2, 3L6J and 5MNW. Other relevant data are available from the corresponding author. Source data are provided with this paper.

Received: 29 January 2021; Accepted: 18 August 2021;

Published online: 17 September 2021

\section{References}

1. Hollenberg, S. M. \& Cinel, I. Bench-to-bedside review: nitric oxide in critical illness-update 2008. Crit. Care 13, 218 (2009).

2. Farah, C., Michel, L. Y. M. \& Balligand, J. L. Nitric oxide signalling in cardiovascular health and disease. Nat. Rev. Cardiol. 15, 292-316 (2018).

3. Bryan, N. S., Bian, K. \& Murad, F. Discovery of the nitric oxide signaling pathway and targets for drug development. Front Biosci. (Landmark Ed.) 14, 1-18 (2009).

4. Sandner, P. From molecules to patients: exploring the therapeutic role of soluble guanylate cyclase stimulators. Biol. Chem. 399, 679-690 (2018).

5. Horst, B. G. \& Marletta, M. A. Physiological activation and deactivation of soluble guanylate cyclase. Nitric Oxide 77, 65-74 (2018).

6. Iyer, L. M., Anantharaman, V. \& Aravind, L. Ancient conserved domains shared by animal soluble guanylyl cyclases and bacterial signaling proteins. BMC Genom. 4, 5 (2003).

7. Kang, Y., Liu, R., Wu, J. X. \& Chen, L. Structural insights into the mechanism of human soluble guanylate cyclase. Nature 574, 206-210 (2019).

8. Horst, B. G. et al. Allosteric activation of the nitric oxide receptor soluble guanylate cyclase mapped by cryo-electron microscopy. Elife 8, https:// doi.org/10.7554/eLife.50634 (2019).

9. Derbyshire, E. R. \& Marletta, M. A. Structure and regulation of soluble guanylate cyclase. Annu. Rev. Biochem. 81, 533-559 (2012).

10. Schrammel, A., Behrends, S., Schmidt, K., Koesling, D. \& Mayer, B. Characterization of $1 \mathrm{H}-[1,2,4]$ oxadiazolo[4,3-a]quinoxalin-1-one as a hemesite inhibitor of nitric oxide-sensitive guanylyl cyclase. Mol. Pharmacol. 50, 1-5 (1996).

11. Olesen, S. P. et al. Characterization of NS 2028 as a specific inhibitor of soluble guanylyl cyclase. Br. J. Pharmacol. 123, 299-309 (1998).

12. Zhao, Y. et al. Inhibition of soluble guanylate cyclase by ODQ. Biochem. (Mosc.) 39, 10848-10854 (2000)

13. Ohlstein, E. H., Wood, K. S. \& Ignarro, L. J. Purification and properties of heme-deficient hepatic soluble guanylate cyclase: effects of heme and other factors on enzyme activation by NO, NO-heme, and protoporphyrin IX. Arch. Biochem. Biophys. 218, 187-198 (1982).

14. Stasch, J. P. et al. Targeting the heme-oxidized nitric oxide receptor for selective vasodilatation of diseased blood vessels. J. Clin. Investig. 116, 2552-2561 (2006)

15. Melichar, V. O. et al. Reduced cGMP signaling associated with neointimal proliferation and vascular dysfunction in late-stage atherosclerosis. Proc. Natl Acad. Sci. U. S. A. 101, 16671-16676 (2004).

16. Evgenov, O. V. et al. NO-independent stimulators and activators of soluble guanylate cyclase: discovery and therapeutic potential. Nat. Rev. Drug Discov. 5, 755-768 (2006).

17. Follmann, M. et al. The chemistry and biology of soluble guanylate cyclase stimulators and activators. Angew. Chem. Int. Ed. Engl. 52, 9442-9462 (2013).

18. Dasgupta, A., Bowman, L., D’Arsigny, C. L. \& Archer, S. L. Soluble guanylate cyclase: a new therapeutic target for pulmonary arterial hypertension and chronic thromboembolic pulmonary hypertension. Clin. Pharmacol. Ther. 97, 88-102 (2015).

19. Armstrong, P. W. et al. Vericiguat in Patients with Heart Failure and Reduced Ejection Fraction. N. Engl. J. Med. 382, 1883-1893 (2020).

20. Ko, F. N., Wu, C. C., Kuo, S. C., Lee, F. Y. \& Teng, C. M. YC-1, a novel activator of platelet guanylate cyclase. Blood 84, 4226-4233 (1994).

21. Mittendorf, J. et al. Discovery of riociguat (BAY 63-2521): a potent, oral stimulator of soluble guanylate cyclase for the treatment of pulmonary hypertension. Chemmedchem 4, 853-865 (2009).

22. Becker, E. M. et al. NO-independent regulatory site of direct sGC stimulators like YC-1 and BAY 41-2272. BMC Pharmacol. 1, 13 (2001).

23. Stasch, J. P. et al. NO-independent regulatory site on soluble guanylate cyclase. Nature 410, 212-215 (2001)

24. Lamothe, M., Chang, F. J., Balashova, N., Shirokov, R. \& Beuve, A. Functional characterization of nitric oxide and YC-1 activation of soluble guanylyl cyclase: structural implication for the YC-1 binding site? Biochemistry 43 3039-3048 (2004).

25. Yazawa, S., Tsuchiya, H., Hori, H. \& Makino, R. Functional characterization of two nucleotide-binding sites in soluble guanylate cyclase. J. Biol. Chem. 281, 21763-21770 (2006)

26. Agullo, L., Buch, I., Gutierrez-de-Teran, H., Garcia-Dorado, D. \& Villa-Freixa, J. Computational exploration of the binding mode of heme-dependent stimulators into the active catalytic domain of soluble guanylate cyclase. Proteins 84, 1534-1548 (2016).

27. Mota, F., Allerston, C. K., Hampden-Smith, K., Garthwaite, J. \& Selwood, D. L. Surface plasmon resonance using the catalytic domain of soluble guanylate cyclase allows the detection of enzyme activators. Bioorg. Med. Chem. Lett. 24 1075-1079 (2014)

28. Denninger, J. W. et al. Interaction of soluble guanylate cyclase with YC-1: kinetic and resonance Raman studies. Biochemistry 39, 4191-4198 (2000).

29. Purohit, R. et al. YC-1 binding to the beta subunit of soluble guanylyl cyclase overcomes allosteric inhibition by the alpha subunit. Biochem. (Mosc.) 53, 101-114 (2014).

30. Wales, J. A. et al. Discovery of stimulator binding to a conserved pocket in the heme domain of soluble guanylyl cyclase. J. Biol. Chem. 293, 1850-1864 (2018).

31. Roy, B., Halvey, E. J. \& Garthwaite, J. An enzyme-linked receptor mechanism for nitric oxide-activated guanylyl cyclase. J. Biol. Chem. 283, 18841-18851 (2008).

32. Friebe, A., Schultz, G. \& Koesling, D. Sensitizing soluble guanylyl cyclase to become a highly CO-sensitive enzyme. EMBO J. 15, 6863-6868 (1996).

33. Derbyshire, E. R. \& Marletta, M. A. Butyl isocyanide as a probe of the activation mechanism of soluble guanylate cyclase. Investigating the role of non-heme nitric oxide. J. Biol. Chem. 282, 35741-35748 (2007).

34. Stasch, J. P. et al. Pharmacological actions of a novel NO-independent guanylyl cyclase stimulator, BAY 41-8543: in vitro studies. Br. J. Pharmacol. 135, 333-343 (2002).

35. Kharitonov, V. G., Sharma, V. S., Magde, D. \& Koesling, D. Kinetics and equilibria of soluble guanylate cyclase ligation by CO: effect of YC-1. Biochem. (Mosc.) 38, 10699-10706 (1999).

36. $\mathrm{Hu}, \mathrm{X}$. et al. Allostery in recombinant soluble guanylyl cyclase from Manduca sexta. J. Biol. Chem. 283, 20968-20977 (2008).

37. Stasch, J. P. et al. NO- and haem-independent activation of soluble guanylyl cyclase: molecular basis and cardiovascular implications of a new pharmacological principle. Br. J. Pharmacol. 136, 773-783 (2002).

38. Martin, F. et al. Structure of cinaciguat (BAY 58-2667) bound to Nostoc H-NOX domain reveals insights into heme-mimetic activation of the soluble guanylyl cyclase. J. Biol. Chem. 285, 22651-22657 (2010).

39. Tsai, A. L. et al. Is Nostoc H-NOX a NO sensor or redox switch? Biochem. (Mosc.) 49, 6587-6599 (2010)

40. Surmeli, N. B. \& Marletta, M. A. Insight into the rescue of oxidized soluble guanylate cyclase by the activator cinaciguat. Chembiochem 13, 977-981 (2012).

41. Schmidt, P., Schramm, M., Schroder, H. \& Stasch, J. P. Preparation of hemefree soluble guanylate cyclase. Protein Expr. Purif. 31, $42-46$ (2003).

42. Wedel, B. et al. Mutation of His-105 in the beta 1 subunit yields a nitric oxideinsensitive form of soluble guanylyl cyclase. Proc. Natl Acad. Sci. USA 91 2592-2596 (1994).

43. Foerster, J., Harteneck, C., Malkewitz, J., Schultz, G. \& Koesling, D. A functional heme-binding site of soluble guanylyl cyclase requires intact $\mathrm{N}$-termini of alpha 1 and beta 1 subunits. Eur. J. Biochem. 240, 380-386 (1996).

44. Rekowski, M. V. W. et al. Insights into soluble guanylyl cyclase activation derived from improved heme-mimetics. J. Med. Chem. 56, 8948-8952 (2013)

45. Childers, K. C. et al. Synergistic mutations in soluble guanylyl cyclase (sGC) reveal a key role for interfacial regions in the sGC activation mechanism. $J$. Biol. Chem. 294, 18451-18464 (2019).

46. Ruiz-Stewart, I. et al. Guanylyl cyclase is an ATP sensor coupling nitric oxide signaling to cell metabolism. Proc. Natl Acad. Sci. U. S. A. 101, 37-42 (2004).

47. Cary, S. P., Winger, J. A. \& Marletta, M. A. Tonic and acute nitric oxide signaling through soluble guanylate cyclase is mediated by nonheme nitric oxide, ATP, and GTP. Proc. Natl Acad. Sci. U. S. A. 102, 13064-13069 (2005)

48. Tsai, A. L., Berka, V., Sharina, I. \& Martin, E. Dynamic ligand exchange in soluble guanylyl cyclase (sGC): implications for sGC regulation and desensitization. J. Biol. Chem. 286, 43182-43192 (2011)

49. Stone, J. R. \& Marletta, M. A. Soluble guanylate cyclase from bovine lung: activation with nitric oxide and carbon monoxide and spectral characterization of the ferrous and ferric states. Biochemistry 33, 5636-5640 (1994).

50. Zheng, S. Q. et al. MotionCor2: anisotropic correction of beam-induced motion for improved cryo-electron microscopy. Nat. Methods 14, 331-332 (2017)

51. Zhang, K. Gctf: real-time CTF determination and correction. J. Struct. Biol. 193, 1-12 (2016)

52. Zivanov, J. et al. New tools for automated high-resolution cryo-EM structure determination in RELION-3. eLife 7, 42166 (2018).

53. Punjani, A., Rubinstein, J. L., Fleet, D. J. \& Brubaker, M. A. cryoSPARC: algorithms for rapid unsupervised cryo-EM structure determination. Nat. Methods 14, 290-296 (2017).

54. Chen, S. et al. High-resolution noise substitution to measure overfitting and validate resolution in 3D structure determination by single particle electron cryomicroscopy. Ultramicroscopy 135, 24-35 (2013). 
55. Pettersen, E. F. et al. UCSF Chimera-a visualization system for exploratory research and analysis. J. Comput. Chem. 25, 1605-1612 (2004).

56. Emsley, P., Lohkamp, B., Scott, W. G. \& Cowtan, K. Features and development of Coot. Acta Crystallogr. D. Biol. Crystallogr. 66, 486-501 (2010).

57. Adams, P. D. et al. PHENIX: a comprehensive Python-based system for macromolecular structure solution. Acta Crystallogr. D. Biol. Crystallogr. 66, 213-221 (2010).

58. Pettersen, E. F. et al. UCSF ChimeraX: structure visualization for researchers, educators, and developers. Protein Sci. https://doi.org/10.1002/pro.3943 (2020).

\section{Acknowledgements}

We thank all of Chen Lab members for kindly help. Cryo-EM data collection was supported by Electron microscopy laboratory and Cryo-EM platform of Peking University with the assistance of Xuemei Li, Zhenxi Guo, Bo Shao, Xia Pei and Guopeng Wang. Part of the structural computation was also performed on the Computing Platform of the Center for Life Science and High-performance Computing Platform of Peking University. We thank the National Center for Protein Sciences at Peking University in Beijing, China for assistance with negative stain EM. The work is supported by grants from National Natural Science Foundation of China (91957201, 31870833 and 31821091 L.C.). Y.K. is supported by the Boya Postdoctoral Fellowship of Peking University.

\section{Author contributions}

L.C. initiated the project. R.L. purified protein and prepared the cryo-EM sample. R.L. and Y.K. collected and processed the cryo-EM data, built and refined the model, and did the enzymatic activity assay. All authors contributed to the paper preparation.

\section{Competing interests}

The authors declare no competing interests.

\section{Additional information}

Supplementary information The online version contains supplementary material available at https://doi.org/10.1038/s41467-021-25617-0.

Correspondence and requests for materials should be addressed to Lei Chen.

Peer review information Nature Communications thanks the anonymous reviewers for their contributions to the peer review of this work.

Reprints and permission information is available at http://www.nature.com/reprints

Publisher's note Springer Nature remains neutral with regard to jurisdictional claims in published maps and institutional affiliations.

\section{(c) (i)}

Open Access This article is licensed under a Creative Commons Attribution 4.0 International License, which permits use, sharing, adaptation, distribution and reproduction in any medium or format, as long as you give appropriate credit to the original author(s) and the source, provide a link to the Creative Commons license, and indicate if changes were made. The images or other third party material in this article are included in the article's Creative Commons license, unless indicated otherwise in a credit line to the material. If material is not included in the article's Creative Commons license and your intended use is not permitted by statutory regulation or exceeds the permitted use, you will need to obtain permission directly from the copyright holder. To view a copy of this license, visit http://creativecommons.org/ licenses/by/4.0/.

(C) The Author(s) 2021 